\title{
Circuit Modelling and Eigenfrequency Analysis of a Poly-Si Based RF MEMS Switch Designed and Modelled for IEEE 802.11 ad Protocol
}

\author{
Tejinder Singh* \\ Discipline of Electronics and Electrical Engineering, Lovely Professional University, Phagwara, Punjab, India \\ tejinder.singh@ieee.org
}

Farzaneh Pashaie

Department of Mechatronics, Islamic Azad University, South Branch, Tehran, Iran

st_f_pashaie@azad.ac.ir

\begin{abstract}
This paper presents the equivalent circuit modelling and eigenfrequency analysis of a wideband robust capacitive radio frequency (RF) microelectromechanical system (MEMS) switch that was designed using Poly-Si and Au layer membrane for highly reliable switching operation. The circuit characterization includes the extraction of resistance, inductance, on and off state capacitance, and $Q$-factor. The first six eigenfrequencies are analyzed using a finite element modeler, and the equivalent modes are demonstrated. The switch is optimized for millimeter wave frequencies, which indicate excellent RF performance with isolation of more than $55 \mathrm{~dB}$ and a low insertion loss of $0.1 \mathrm{~dB}$ in the V-band. The designed switch actuates at $13.2 \mathrm{~V}$. The $R, L, C$ and $Q$-factor are simulated using $Y$-matrix data over a frequency sweep of 20-100 GHz. The proposed switch has various applications in satellite communication networks and can also be used for devices that will incorporate the upcoming IEEE Wi-Fi 802.11ad protocol.
\end{abstract}

Category: Smart and intelligent computing

Keywords: RF MEMS; Equivalent circuit modelling; Eigenfrequency analysis; Capacitive switch; Millimeter wave frequencies

\section{INTRODUCTION}

In the last decade, various efforts have been made for radio frequency (RF) microelectromechanical system (MEMS) switches that are applicable to devices with switching activities that operate in RF to millimeter wave frequencies. MEMSs that are especially for RF applications have captured the interest of researchers from aca- demia and industry. The RF MEMS has various advantages like high isolation, low insertion loss, low or near-zero power consumption, and low intermodulation distortion over their semiconductor counterparts like the field-effect transistor and p-i-n diode switches [1-5]. MEMS switches suffer from some drawbacks like low switching speed (due to mechanical deflection), high electrostatic voltage requirements of around $20-60 \mathrm{~V}$ for reliable switching

This is an Open Access article distributed under the terms of the Creative Commons Attribution Non-Commercial License (http://creativecommons.org/licenses/ by-nc/3.0/) which permits unrestricted non-commercial use, distribution, and reproduction in any medium, provided the original work is properly cited. 
operation, low power handling capability, reliability issues like average lifetime, fracture concerns, and packaging constraints [6-8]. RF MEMS switches incorporate a thin movable metal membrane, which actuates electrostatically for switching operations [9]. The lifetime of MEMS switches is one of the primary concerns for their use in repair-free applications like in remote places. After a few million switching cycles the metal membrane starts to suffer from fractures and this breakage usually occurs at high stress areas of the thin metal membrane.

In this paper, we present the circuit characterization and eigenfrequency analysis of a wideband robust capacitive RF MEMS switch design that incorporates a thinlayered membrane of Poly-Si and Au millimeter wave frequencies. The switch design we present is simulated using commercially available EM solvers. We present the mechanical modelling viz. stress analysis that is reported in detail in [10], and the analysis using different proportions of materials is also reported in [10]. We numerically calculate the force distribution and equivalent circuit parameters of the RF MEMS switch. The basic model is taken as reference [9], and various components like the $R$, $L, C, Q$-factor, and $S$-parameters are computed using the data of a $Y$-matrix, over a frequency sweep of 20-100 GHz. The first six eigenfrequencies are analyzed, and the equivalent modes are presented. The extracted and fitted $R L C$ components of this switch over a range of frequencies divided into different frequency bands are also summarized in Section III. The proposed switch has huge potential for use in applications that operate on millimeter waves like satellite communication networks.

\section{DESIGN AND PRINCIPLE OF OPERATION}

The operational principle of the capacitive switch is mentioned in [11]. RF MEMS are usually actuated by the electrostatic principle [6]. For reliability and robustness of the switch, it is mandatory to design the switch with thick meanders to get an optimized range between the elastic recovery forces of metal and electrostatic voltage requirements for switching operation. The most common reliability issue in RF MEMS like stiction can be reduced, if the elastic recovery forces of the membrane are higher [12]. To overcome this issue of stiction, we have proposed a twin-layer membrane based on Gold $(\mathrm{Au})$ and Polysilicon (Poly-Si). Gold has excellent electrical conductivity, but has low stress handling capabilities; in contrast, Poly$\mathrm{Si}$ is a semiconductor, but has very high ultimate tensile strength to increase reliability. Gold $(\mathrm{Au})$ is commonly used as a material for membrane design, but its ultimate tensile stress is $100 \mathrm{MPa}$, i.e., if the stress gradient crosses $100 \mathrm{MPa}$, the membrane can easily break from the areas under high stress. In contrast, the tensile strength of Poly$\mathrm{Si}$ is $1.2 \mathrm{GPa}$ [13] with a high Young's modulus; thus using Poly-Si alone can increase the actuation voltage requirements

This switch incorporates a thin membrane of layered $\mathrm{Au}$ and Poly-Si. A layer of $0.2 \mu \mathrm{m}$ thick Poly-Si is combined on top of a $0.5 \mu \mathrm{m}$ thick Au layer. This combination provides high elastic recovery to reduce stiction and makes the switch more robust to handle many more switching cycles.

The switch is designed on quartz substrate due to its better EM wave propagation capabilities on higher frequency bands [14]. A coplanar waveguide (CPW) with wide signal line of $60 \mu \mathrm{m}$ and $40 \mu \mathrm{m}$ gap between the ground plane is designed over the substrate to allow or block the RF signal using thin membrane. Input and output ports of the signal line are usually coupled with coaxial cables to connect with standard RF applications. Au material is used to design the CPW due to its better stiction with silicon nitride as dielectric over the substrate. Fig. 1 shows the proposed RF MEMS switch with labeled components. When the bias voltage is provided to the electrode beneath the signal line, electrostatic force is generated, which causes vertical deflection towards the signal line. A dielectric layer of $0.2 \mu \mathrm{m}$ thick is used between the membrane and signal line contact to change the value of the capacitance.

The capacitance varies either to allow the RF signal or to pass it through ground, i.e., block the RF signal. A large electrode of $80 \mu \mathrm{m} \times 60 \mu \mathrm{m}$ is used for the actuation principle. The meanders help in reducing the pulldown voltage. Au is used to achieve low-voltage and Poly-Si is used for increasing robustness of the switch; hence, the best of both materials makes this switch reliable in high frequency applications. Fig. 2 demonstrates a close-up view of the membrane that shows twin layers stacked on each other. Holes are provided in the area of membrane that usually interacts with the signal line for the switching operation. Inclusion of holes in the membrane has huge advantages - it aids in attaining higher switching speeds, reduction of biaxial residual stress, reduction in air damping, and the effective mass of the membrane decreases [9]. The dimensions of the membrane are shown in Fig. 3 and the equivalent circuit model of the switch is shown in Fig. 4. The $R, L$, and $C$

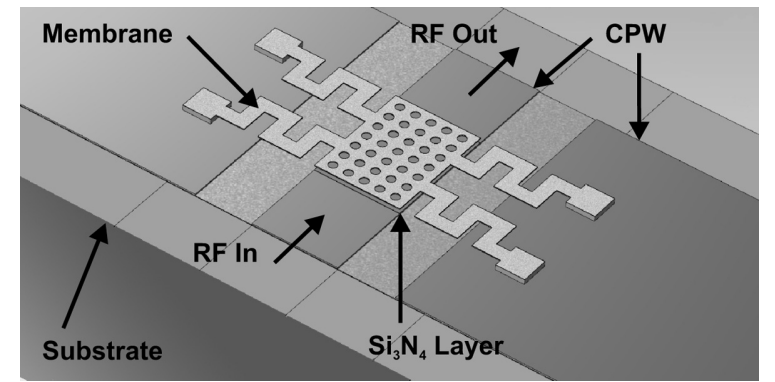

Fig. 1. Switch geometry of the proposed radio frequency (RF) microelectromechanical system switch demonstrating membrane over coplanar waveguide (CPW) and $\mathrm{Si}_{3} \mathrm{~N}_{4}$ dielectric layer. 


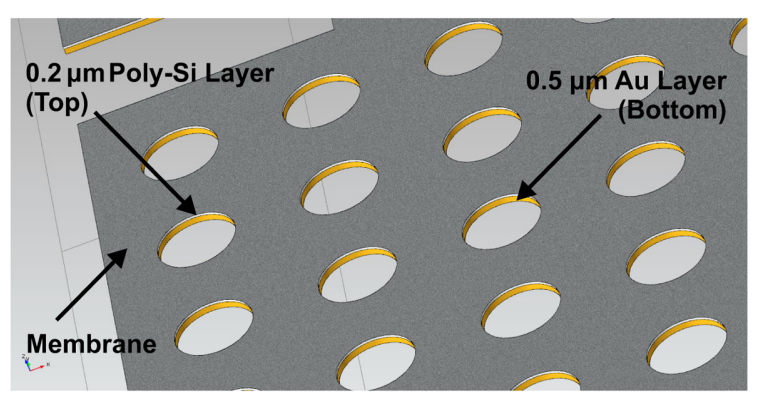

Fig. 2. Close-up view of layered Poly-Si and Au membrane, the image showing the $0.2 \mu \mathrm{m}$ Poly-Si layer on top of $0.5 \mu \mathrm{m}$ thick gold layer. The image shown is a close-up view taken from the corner area of the central part of membrane.

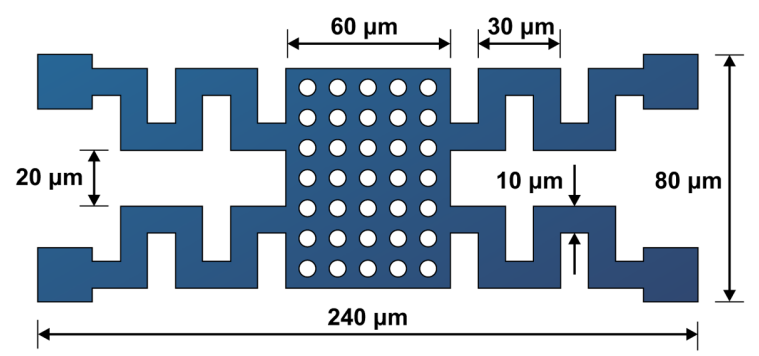

Fig. 3. Dimensions of layered switch membrane; the complete membrane acquires area of $240 \mu \mathrm{m} \times 80 \mu \mathrm{m}$.

components are in shunt configuration of transmission as the switch is a shunt capacitive RF MEMS switch. The capacitance is a variable component in the shunt line. This variable component changes its value during switching. The specifications of the designed switch are given in Table 1.

\section{CIRCUIT CHARACTERIZATION}

The basic elements $R, L$, and $C$ are extracted using commercially available EM-solvers [15]. The extracted values of these components are given in Table 2 for different frequency bands from 20-100 GHz. As the switch is a capacitive switch, the capacitance for ON-state or upstate and OFF or down-state is extracted. The corre-

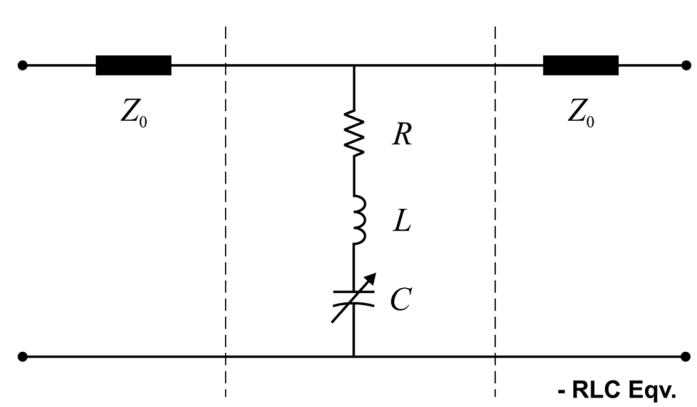

Fig. 4. Equivalent $C L R$ circuit model of proposed switch. The circuit is shown for the shunt configuration of switch. $Z_{0}$ are the terminal impedances and component $C$ is variable, the value of $C$ is varied by the position of metal membrane over the signal line.

Table 1. Switch specifications

\begin{tabular}{lcccc}
\hline \multicolumn{1}{c}{ Component } & L & W & D & Material \\
\hline Substrate & 540 & 100 & 150 & Quartz \\
Dielectric & 540 & 100 & 0.5 & $\mathrm{Si}_{3} \mathrm{~N}_{4}$ \\
CPW (G S G) & 40 & 60 & 1.0 & $\mathrm{Gold}$ \\
Tx - Dielectric & 60 & 40 & 0.2 & $\mathrm{Si}_{3} \mathrm{~N}_{4}$ \\
Beam bottom & 240 & 80 & 0.5 & Gold \\
Beam top & 240 & 80 & 0.2 & Poly-Si \\
Meanders & 70 & 10 & 0.7 & Poly-Si \\
Meanders gap & 10 & 10 & $\mathrm{NA}$ & - \\
Hole size & 6 & 6 & 0.7 & - \\
Gap, $g_{0}$ & - & - & 3 & - \\
Anchors & 20 & 20 & 4 & Gold \\
\hline Allits are in & & & &
\end{tabular}

All units are in $\mu \mathrm{m}$.

CPW: coplanar waveguide, NA: not available.

sponding fitted values are also given in Table 2 . The table is summarized for the extracted and fitted value of $R, L$, and $C$ for $60 \mathrm{GHz}, \mathrm{V}$-band and for different frequency ranges. The switch performance is excellent around $60 \mathrm{GHz}$. The exact fit is computed according to the $E$-standards. The extracted capacitance in both states of switch for complete frequency sweep is shown in Fig. 5, and Fig. 6

Table 2. Extracted and fitted values

\begin{tabular}{|c|c|c|c|c|c|c|c|c|c|c|}
\hline \multirow{2}{*}{$\begin{array}{l}\text { Frequency } \\
\text { range }\end{array}$} & \multicolumn{4}{|c|}{ Extracted } & \multicolumn{4}{|c|}{ Fitted } & \multicolumn{2}{|c|}{$Q$-factor } \\
\hline & $C_{u p}(\mathrm{fF})$ & $C_{\text {down }}(\mathrm{pF})$ & $R(\Omega)$ & $L(\mathrm{pH})$ & $C_{u p}(\mathbf{f F})$ & $C_{\text {down }}(\mathrm{pF})$ & $R(\Omega)$ & $L(\mathrm{pH})$ & ON & OFF \\
\hline V-band & 26.1 & 1.51 & 0.56 & 96.5 & 27 & 1.5 & 0.56 & 100 & 27.2 & 19.3 \\
\hline 60 & 25.6 & 1.52 & 0.59 & 98.1 & 27 & 1.5 & 0.56 & 100 & 28.5 & 18.3 \\
\hline $40-85$ & 26.2 & 1.20 & 0.57 & 92.7 & 27 & 1.2 & 0.56 & 91 & 27.0 & 16.9 \\
\hline $20-100$ & 27.0 & 1.10 & 0.56 & 88.2 & 27 & 1.1 & 0.56 & 91 & 26.3 & 15.8 \\
\hline $80-100$ & 28.8 & 1.51 & 0.45 & 84.8 & 30 & 1.5 & 0.43 & 82 & 21.2 & 21.1 \\
\hline
\end{tabular}




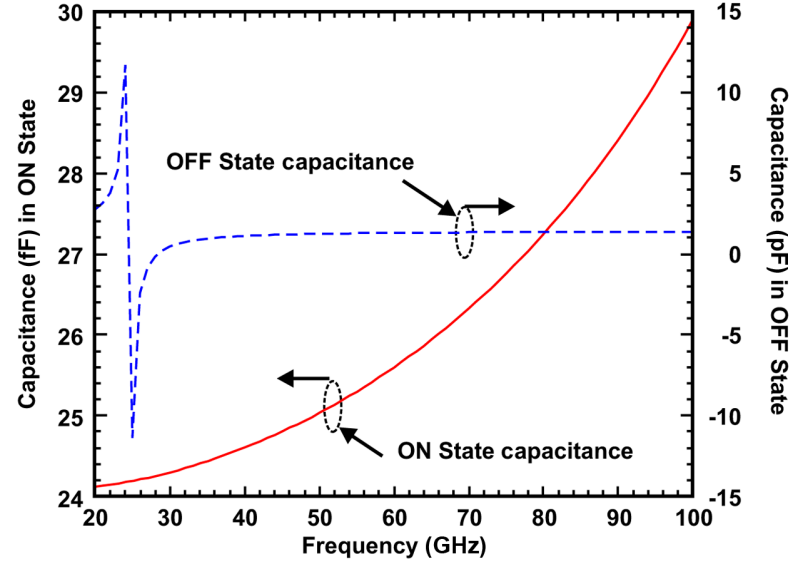

Fig. 5. Capacitance in ON and OFF states of the switch that results in $\approx 27 \mathrm{fF}$ capacitance in ON state and variable according to the input frequency.

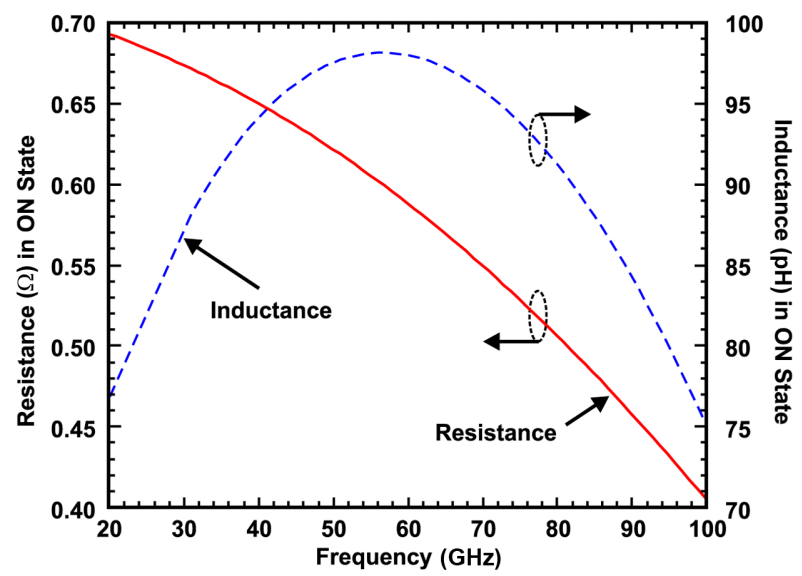

Fig. 6. Simulation result of resistance $(\Omega)$ and inductance $(\mathrm{pH})$ of the MEMS switch, the inductance is high from the $40-80 \mathrm{GHz}$ frequency range, and resistance drops almost linearly as per the input frequency, but lies between $0.7-0.4 \Omega$.

indicates the series resistance and inductance. The $Q$-factor for ON and OFF states is also computed and is shown in Fig. 7.

For the capacitive RF MEMS switch in shunt configuration between signal line and ground, the impedance $Z_{s}$ is given by:

$$
Z_{s}=R_{s}+j \omega L+\frac{1}{j \omega C}
$$

The impedance $Z_{s}$ can also be approximated from the $Y$-matrix data as:

$$
Z_{s}=\frac{-2}{Y_{12}+Y_{21}}
$$

The value of the capacitance depends purely on the position of the metal membrane suspended over the sig-

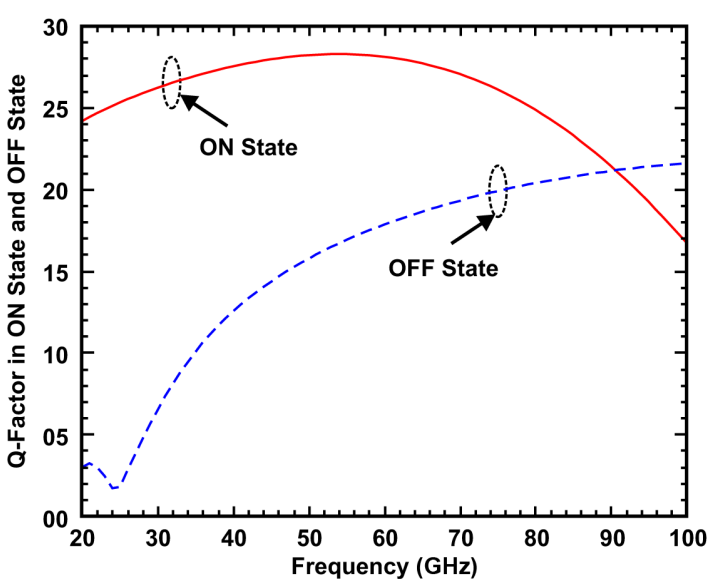

Fig. 7. Q-Factor in ON and OFF state of switch results in a great $Q$-factor of $\approx 25$ in ON state and varies between $5-22$ in OFF state.

nal line. The capacitance depends on the ON or OFF state of the switch. The up-state capacitance $C_{u p}$ for the switch in ON state and the down-state capacitance $C_{\text {down }}$ for the switch in OFF state can be computed by plugging the values into Eqs. (3) and (4):

$$
\begin{gathered}
C_{u p}=\frac{\varepsilon_{0} w W}{g_{0}+\left(t_{d} / \varepsilon_{r}\right)} \\
C_{d o w n}=\frac{\varepsilon_{0} \varepsilon_{r} w W}{t_{d}}
\end{gathered}
$$

where, $\varepsilon_{0}$ is the permittivity of free space, $w$ and $W$ are the length and width of the actuation electrode, respectively, $g_{0}$ is the gap between the switch membrane and signal line, $t_{d}$ is the thickness of the dielectric layer, and $\varepsilon_{r}$ is the permittivity of the material. The up-state capacitance $C_{u p}$ is $27 \mathrm{fF}$ and the down-state capacitance $C_{\text {down }}$ is $1.4 \mathrm{pF}$.

The primary factors for the calculation of the series resistance $R_{s}$ are due to the transmission line loss, and also due to the membrane of the switch. The series resistance $R_{s}$ is given as:

$$
\alpha=\frac{R_{s} / l}{2 Z_{0}}
$$

where, $l$ is the length of beam, and $Z_{0}$ is the characteristic impedance of the transmission line.

$R_{s}=\operatorname{Re}\left(Z_{s}\right)$ can also be used to estimate the series resistance $R_{s}$ from the $Y$-matrix.

The inductance $L$ can be approximated from the $Y$ matrix data. The value of the impedance $Z_{s}$ is required from Eq. (2) to compute the inductance $L$ as:

$$
L=\frac{\operatorname{Im}\left(Z_{s}\right)}{2 \pi f}
$$

A high $Q$-factor is desirable for any electronic switches, 
components or devices. Generally, a $Q$-factor of above 15 is considered good for a variety of RF MEMS switches [9]. This proposed switch has excellent $Q$-factors of above 27 in the ON state and above 15 in the OFF state as shown in Fig. 7. The $Q$-factor can be computed for the $\mathrm{ON}$ and OFF states of the switch as:

$$
Q=\left|\frac{\operatorname{Im}\left(Y_{11}\right)}{\operatorname{Re}\left(Y_{11}\right)}\right|
$$

where, $\operatorname{Im}\left(Y_{11}\right)$ and $\operatorname{Re}\left(Y_{11}\right)$ are the imaginary and real part of the two-port network, respectively, as shown in Fig. 4.

The actuation voltage $V_{p}$ required to actuate the switching mechanism can be computed using:

$$
V_{p}=\sqrt{\frac{8 k}{27 \varepsilon_{0} w W} g_{0}^{3}}
$$

where, $k$ is the spring constant and can be determined using Eq. (10), $w$ is the length of electrode, $W$ is the width of electrode, and $g_{0}$ is the gap between signal line and membrane. The calculated $V_{p}$ for this switch is $13.2 \mathrm{~V}$.

\section{EIGENFREQUENCY ANALYSIS}

Eigenfrequency basically means the 'characteristic' frequency of a device or in simple words, it is the 'ownfrequency' of the device. It can also be thought of as the normal modes of vibrations of a system. Eigenfrequency analysis was used to find the basic modes of vibration of the RF MEMS switch [14]. The first six modes of the switch are shown in Fig. 8. These frequencies can be determined by solving the Lagrangian of the system, and then applying the canonical equation. The deflection at the natural frequency of the RF MEMS switch or the first mode can be determined from Fig. 8. The series of next
Table 3. Eigenfrequencies and membrane deflection

\begin{tabular}{cc}
\hline Mode & Eigenfrequency $(\mathrm{Hz})$ \\
\hline Mode 1 & $30419.406941(30.4 \mathrm{kHz})$ \\
Mode 2 & $47039.669441(47.0 \mathrm{kHz})$ \\
Mode 3 & $81281.166386(81.3 \mathrm{kHz})$ \\
Mode 4 & $1.268 \times 10^{5}(1.27 \mathrm{MHz})$ \\
Mode 5 & $1.633 \times 10^{5}(1.63 \mathrm{MHz})$ \\
Mode 6 & $1.772 \times 10^{5}(1.77 \mathrm{MHz})$ \\
\hline
\end{tabular}

frequencies are at values that are whole number multiples of the fundamental frequency. The first six eigenfrequencies are given in Table 3.

The equation of motion of a membrane that is subjected to harmonic force is considered. The harmonic motion of the mass-spring system in a single degree of freedom is usually modelled with a differential equation of second-order as:

$$
m_{\mathrm{eff}} \frac{d^{2} x}{d t^{2}}+\gamma_{\mathrm{eff}} \frac{d x}{d t}+k_{\mathrm{eff}} x=F_{e}
$$

where, $m_{\text {eff }}$ is the effective mass of the membrane, $\gamma_{\text {eff }}$ is the effective damping coefficient of the dielectric material, $k_{\text {eff }}$ is the effective spring constant, and $F_{e}$ is the electrostatic force. $m_{\text {eff }}$ is composed of Au effective mass $m_{\text {eff } 1}=60.47 \mathrm{ng}$ and Poly-Si effective mass $m_{\mathrm{eff}_{2}}=4.63 \mathrm{ng}$, respectively, for the designed membrane. Hence, the total $m_{\text {eff }}$ is $m_{\text {eff }_{1}}+m_{\text {eff }_{2}}$ that leads to $m_{\text {eff }}=65.1 \mathrm{ng}$. The effective spring constant $k_{\text {eff }}$ is composed of $k^{\prime}$ and $k^{\prime \prime}$. According to [9], the biaxial residual stress component $k^{\prime \prime}$ can be neglected due to the serpentine design, holes to release the biaxial residual stress, and the use of Poly-Si (as the stiffness of Poly-Si quite high). $\gamma_{\text {eff }}$ can be determined by computing Eq. (9) by plugging all remaining values.

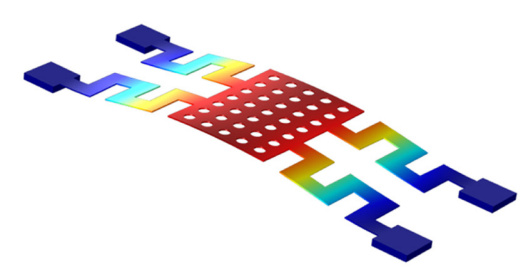

Mode 1

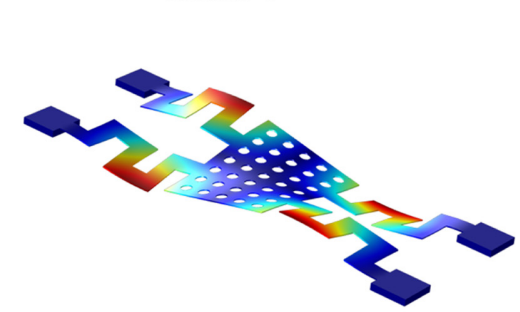

Mode 4

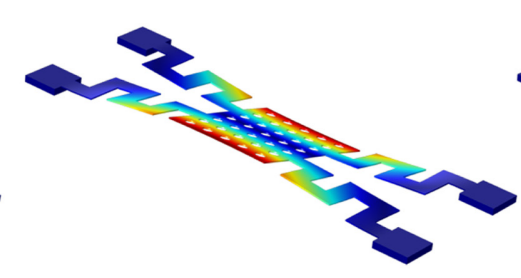

Mode 2

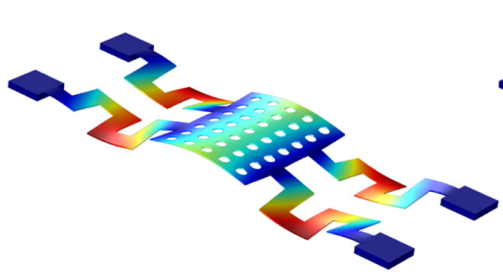

Mode 5

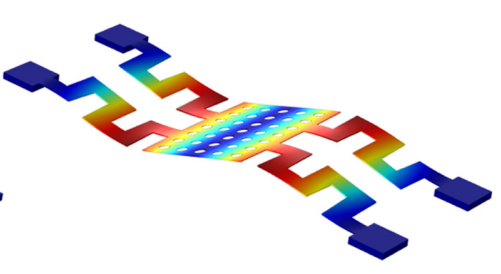

Mode 3

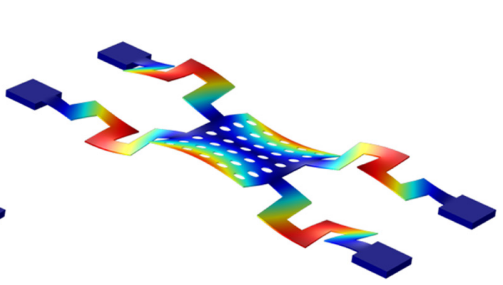

Mode 6

Fig. 8. First six eigenmodes of designed membrane. 
As the membrane is composed of two materials, the spring constant $k^{\prime}$ is further composed of two components: $k_{a}$ for the Au material and $k_{p}$ for the Poly-Si. The spring constant can be computed using:

$$
k^{\prime} \approx \frac{48 G J}{l_{a}^{2}\left(\frac{G J}{E I_{x}} l_{a}+l_{b}\right) n^{3}} \text { for } n » \frac{3 l_{b}}{\frac{G J}{E I_{x}} l_{a}+l_{b}}
$$

where, $n$ is the number of meanders in the serpentine configuration, $G=E / 2(1+v)$ is the torsion modulus, $I_{x}=w t^{3} / 12$ is the moment of inertia and the torsion constant is usually given by:

$$
J=\frac{1}{3} t^{3} w\left(1-\frac{192}{\pi^{5}} \frac{t}{w} \sum_{\substack{t=1 \\ t=\text { odd }}}^{\infty} \frac{1}{i^{5}} \tanh \left(\frac{i \pi w}{2 t}\right)\right)
$$

The effective spring constant $k_{\text {eff }}$ for both components, i.e., $k_{a}+k_{p}$, can be determined by plugging material values like the Young's modulus $E$ and moment of inertia $I_{x}$ into Eq. (10). The combined spring constant $k_{\text {eff }}$ for the membrane is $5.1 \mathrm{~N} / \mathrm{m}$.

The natural frequency of the membrane depends on the equivalent spring constant and the effective mass, and the natural frequency $f_{0}$ is given as:

$$
f_{0}=\frac{1}{2 \pi} \sqrt{\frac{k_{\text {eff }}}{m}}
$$

To determine the first mode of eigenfrequency $f_{1}$, the component needs to be computed first for $\mathrm{Au}$ and then for Poly-Si. Then, the addition of these two factors can give the best estimate of the desired frequency. The first mode of frequency $f_{1}$ can be determined by:

$$
f_{1}=\frac{1}{2 \pi}\left[\frac{15.418}{L^{2}}\right] \sqrt{\frac{E I_{x}}{\rho}}
$$

where, $\rho$ is the mass density (mass/length) and $L$ is the length of membrane.

\section{RF PERFORMANCE ANALYSIS}

The RF performances for the device in both switching states are extracted by $S$-parameters. The data of the $S$ matrix is computed for the $20-100 \mathrm{GHz}$ frequency range [16]. Usually isolation, return loss in OFF state, insertion loss, and return loss in ON state are all required to examine the performance of switch in the RF region. High isolation and low insertion loss are in high demand in some applications, and that is where RF MEMS switches come into the picture. The proposed design gives excellent RF performance, with low voltage requirements. The switch is optimized for wideband millimeter wave frequencies. The $S$-parameters $S_{11}, S_{21}$ in both switching states can be computed using the extracted $Y$ - and $Z$-matrix data by plugging the values in the equations given below. To compute the parameters in ON state, $Y_{11-x}$ will be used, where $x=1$ for ON state and $x=0$ for OFF state.

$$
S_{11-x}=\left(\frac{Y_{z}^{2}-Y_{11-x}^{2}+Y_{21-x}^{2}}{\left(Y_{11-x}+Y_{z}\right)^{2}-Y_{21-x}^{2}}\right)
$$

where, $S_{11-x}$ is the return loss on ON or OFF state depending on the variable $x, Y_{z}=1 / Z_{0}, Y_{11-x}=j \omega C_{\text {down }}$ for $x=0$, i.e., in OFF state and $Y_{11-x}=j \omega C_{u p}$ for $x=1$, i.e., in ON state. $Y_{21-x}=-j \omega C_{\text {down }}$ for $x=0$ and $Y_{21-x}=-j \omega C_{u p}$ for $x=$ 1, i.e., in OFF and ON states, respectively. To estimate the isolation $S_{21-0}$ and insertion loss $S_{21-1}$, plug the values into:

$$
S_{21-x}=\left(\frac{-2 Y_{21-x}^{2} Y_{z}}{\left(Y_{11-x}+Y_{z}\right)^{2}-Y_{21-x}^{2}}\right)
$$

Excellent isolation above $50 \mathrm{~dB}$ can be achieved in the V-band and above $40 \mathrm{~dB}$ from $45-85 \mathrm{GHz}$. At $60 \mathrm{GHz}$, the switch results in maximum isolation of $58 \mathrm{~dB}$. The results indicate 0.07 to $0.15 \mathrm{~dB}$ insertion loss in the $\mathrm{ON}$ state from $40-85 \mathrm{GHz}$. The return loss is in between $0.15-0.30 \mathrm{~dB}$ in the OFF state and $25-40 \mathrm{~dB}$ for $85 \mathrm{GHz}$ and $40 \mathrm{GHz}$, respectively. The proposed switch also indicates moderate performance until $100 \mathrm{GHz}$ with $35 \mathrm{~dB}$ of isolation and $0.2 \mathrm{~dB}$ insertion loss. The $S$-parameters in OFF state can be determined from the plot shown in Fig. 9 and the $S$-parameters plot for the ON state is shown in Fig. 10.

Table 4 summarises the simulated RF performance results for the switch at different frequency bands. The RF performance for 40-60 GHz, 60-100 GHz, V-band $(60-75 \mathrm{GHz}), 60 \mathrm{GHz}$, and $45-85 \mathrm{GHz}$ is given in the table. The plots shown in Figs. 9 and 10 indicate the RF performance from $20-100 \mathrm{GHz}$. The typical values for

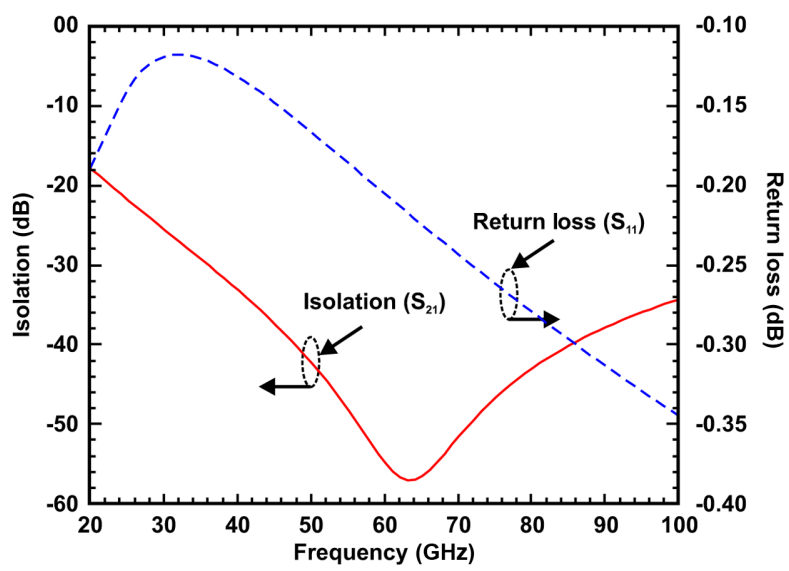

Fig. 9. Simulation of isolation $(\mathrm{dB})$ and return loss $(\mathrm{dB})$ in OFF state of RF MEMS switch. The results demonstrate isolation of $\approx$ $57 \mathrm{~dB}$ at $60 \mathrm{GHz}$ region and less return loss towards higher frequencies. 


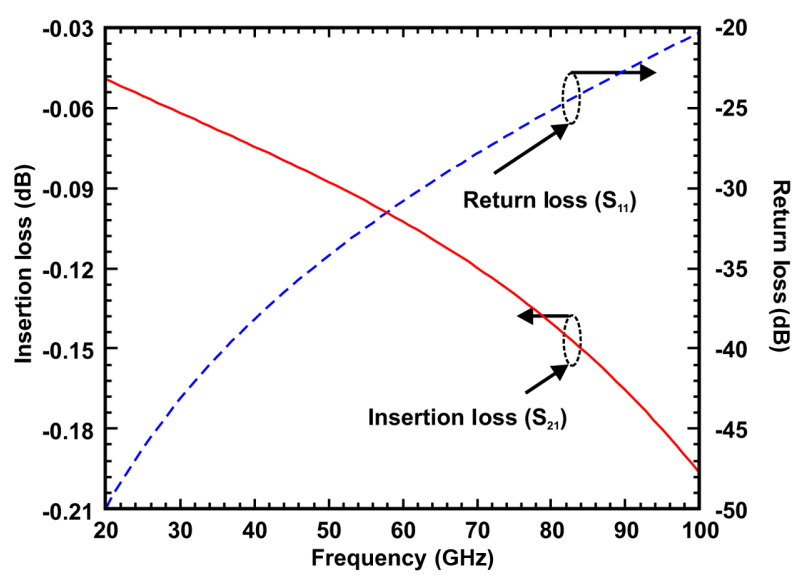

Fig. 10. Simulation of insertion loss $(d B)$ and return loss $(d B)$ plot for the RF MEMS switch in ON state. Excellent insertion loss of $\approx 0.1 \mathrm{~dB}$ and return loss of $\approx 30-40 \mathrm{~dB}$ were observed for 50 $\mathrm{GHz}$ and above.

Table 4. RF performance analysis

\begin{tabular}{lcccc}
\hline $\begin{array}{c}\text { Freq. } \\
\text { range }\end{array}$ & Isolation & $\begin{array}{c}\text { Insertion } \\
\text { loss }\end{array}$ & $\begin{array}{c}\text { Return loss } \\
\text { OFF }\end{array}$ & $\begin{array}{c}\text { Return loss } \\
\text { ON }\end{array}$ \\
\hline $40-60$ & $>30$ & $<0.1$ & $<0.2$ & $>30$ \\
$60-100$ & $>35$ & $<0.2$ & $<0.35$ & $>20$ \\
$45-85$ & $>40$ & $<0.15$ & $<0.3$ & $>30$ \\
60 & $=57$ & $=0.1$ & $=0.22$ & $=32$ \\
V-band & $>55$ & $<0.13$ & $<0.25$ & $>30$
\end{tabular}

All units are in $\mathrm{dB}$.

RF MEMS switches that operate in millimeter wave frequencies usually result in isolation of approximately $20 \mathrm{~dB}$ and insertion loss having typical values of $0.3-$ $0.4 \mathrm{~dB}$, because on moving towards the higher frequency spectrum, the performance start decreasing.

\section{CONCLUSION}

A wideband robust capacitive RF MEMS switch is designed and characterized. The combined layer membrane shows excellent RF performance in millimeter wave frequencies up to $100 \mathrm{GHz}$, while requiring low actuation voltage. The switch incorporates a twin membrane approach to increase the reliability operation of the switch. The high $Q$-factor makes this switch an excellent choice for high-frequency applications. Maximum performance can be achieved at $60 \mathrm{GHz}$; hence, the proposed switch can be used in upcoming IEEE Wi-Fi standard 802.11ad that will operate at $60 \mathrm{GHz}$ with data transfer rate up to $7 \mathrm{Gbps}$, or in cross link communication networks between satellites in a constellation.

\section{ACKNOWLEDGMENTS}

The authors would like to thank Dr. X. Liu and Dr. Prof. G. Wang for their suggestions in circuit modelling, and Lovely Professional University, PB for providing the necessary resources to conduct this research work.

\section{REFERENCES}

1. C. L. Goldsmith, Z. Yao, S. Eshelman, and D. Denniston, "Performance of low-loss RF MEMS capacitive switches," IEEE Microwave and Guided Wave Letters, vol. 8, no. 8, pp. 269-271, 1998.

2. G. M. Rebeiz and J. B. Muldavin, "RF MEMS switches and switch circuits," IEEE Microwave Magazine, vol. 2, no. 4, pp. 59-71, 2001.

3. D. Hyman and M. Mehregany, "Contact physics of gold microcontacts for MEMS switches," IEEE Transactions on Components and Packaging Technologies, vol. 22, no. 3, pp. 357-364, 1999.

4. J. Kennedy, "Surface mount components reduce broadband equipment costs," Applied Microwave and Wireless, vol. 13, no. 1, pp. 102-108, 2001.

5. D. Peroulis, S. Pacheco, K. Sarabandi, and P. B. Katehi, "MEMS devices for high isolation switching and tunable filtering," in Proceedings of the IEEE MTT-S International Microwave Symposium Digest, Boston, MA, 2000, pp. 12171220.

6. S. D. Lee, B. C. Jun, S. D. Kim, H. C. Park, J. K. Rhee, and K. Mizuno, "An RF-MEMS switch with low-actuation voltage and high reliability," Journal of Microelectromechanical Systems, vol. 15, no. 6, pp. 1605-1611, 2006.

7. S. Pacheco, C. T. Nguyen, and L. P. Katehi, "Micromechanical electrostatic K-band switches," in Proceedings of the IEEE MTT-S International Microwave Symposium Digest, Baltimore, MD, 1998, pp. 1569-1572.

8. S. C. Shen, D. Caruth, and M. Feng, "Broadband low actuation voltage RF MEMS switches," in Proceedings of the 22nd IEEE GaAs IC Symposium, Seattle, WA, 2000, pp. 161-164.

9. G. M. Rebeiz, RF MEMS: Theory, Design, and Technology, New York: Wiley, 2003.

10. T. Singh and A. Kumari, "Design and modelling of a robust wideband poly-Si and $\mathrm{Au}$ based capacitive RF MEMS switch for millimeter wave applications," in Proceedings of the 2nd International Conference on Computing Sciences, 2013, pp. 106-114.

11. T. Singh, "Effective stress modelling of membranes made of gold and aluminum materials used in radio-frequency microelectromechanical system switches," Transactions on Electrical and Electronic Materials, vol. 14, no. 4, pp. 172-176, 2013.

12. C. Palego, J. Deng, Z. Peng, S. Halder, J. C. Hwang, D. I. Forehand, D. Scarbrough, C. L. Goldsmith, I. Johnston, S. K. Sampath, et al., "Robustness of RF MEMS capacitive switches with molybdenum membranes," IEEE Transactions on Microwave Theory and Techniques, vol. 57, no. 12, 
pp. 3262-3269, 2009

13. W. N. Sharpe, B. Yuan, R. Vaidyanathan, and R. L. Edwards, "Measurements of Young's modulus, Poisson's ratio, and tensile strength of polysilicon," in Proceedings of the IEEE 10th Annual International Workshop on Micro Electro Mechanical Systems, Nagoya, Japan, 1997, pp. 424429.

14. J. B. Rizk and G. M. Rebeiz, "W-band CPW RF MEMS circuits on quartz substrates," IEEE Transactions on Microwave Theory and Techniques, vol. 51, no. 7, pp. 1857-1862,
2003.

15. J. B. Muldavin and G. M. Rebeiz, "High-isolation CPW MEMS shunt switches. Part 1: modelling," IEEE Transactions on Microwave Theory and Techniques, vol. 48, no. 6, pp. 1045-1052, 2000.

16. T. Singh and N. Khaira, "High isolation single-pole fourthrow RF MEMS switch based on series-shunt configuration," The Scientific World Journal, vol. 2014, no. 605894, pp. 1-6, 2014.

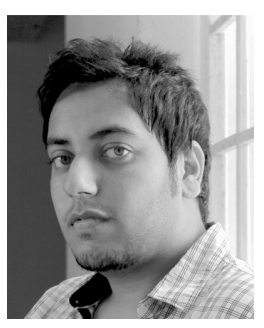

\section{Tejinder Singh}

Tejinder Singh received his Bachelor of Technology degree in Electronics and Communication Engineering from Lovely Professional University, PB in 2010, and Master of Technology degree in VLSI Design from Lovely Professional University, PB in 2014. He has received an IEEE AsiaPacific Region PG Award, the IEEE M. V. Chauhan Award, and various best paper awards. He is currently serving as a reviewer for Transactions on Electrical and Electronic Materials of South Korea, Taylor and Francis International Journal of Electronics, and currently serving the technical program committee of the 11th International Workshop on Mobile Applications in Spain; International Workshop on the Design and Performance of Networks on Chip in Canada; International Journal of Computing and Digital Systems in UK; International Journal of Innovative and Emerging Development in UK; 2014 ICEPIT in Bahrain; and International Conference on Science and Technology for Sustainability in Indonesia. He has published many research papers in peer-reviewed journals and in international conferences. His research interests include the design and characterization of RF MEMS switches, BioMEMS devices, Low-power VLSI circuits, and Memristor/Memristive systems.

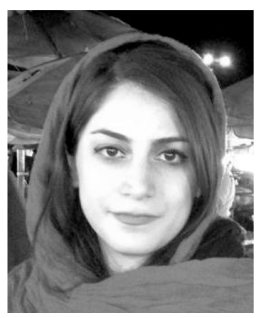

\section{Farzaneh Pashaie}

Farzaneh Pashaie received her Bachelor of Science degree in Electronics Engineering from Islamic Azad University, Saveh Branch, Tehran, Iran in 2010, and Master of Science degree in Mechatronic from Islamic Azad University, South Branch, Tehran, Iran in 2014. She has published many research papers in peerreviewed journals and international conferences. Her research interests include RF MEMS, VLSI circuits and systems, and Mechatronics and brain interfacing electrodes design and characterization using a Multiphysics environment. 